Article

\title{
Feasibility Study of a Proton Irradiation Facility for Radiobiological Measurements at an $18 \mathrm{MeV}$ Cyclotron
}

\author{
Anna Baratto-Roldán 1,2,*(D), María del Carmen Jiménez-Ramos ${ }^{1}{ }^{(0)}$, Maria Cristina Battaglia ${ }^{2}$, \\ Javier García-López ${ }^{1,2}{ }^{\mathbb{D}}$, María Isabel Gallardo ${ }^{2} \mathbb{D}$, Miguel A. Cortés-Giraldo ${ }^{2} \mathbb{D}$ and José $\mathrm{M}$. \\ Espino ${ }^{1,2}$ (1) \\ 1 Centro Nacional de Aceleradores, 41092 Sevilla, Spain; mcyjr@us.es (M.C.J.-R.); fjgl@us.es (J.G.-L.); \\ espino@us.es (J.M.E.) \\ 2 Department of Atomic, Molecular and Nuclear Physics, Universidad de Sevilla, 41012 Sevilla, Spain; \\ mbattaglia@us.es (M.C.B.); gallardo@us.es (M.I.G.); miancortes@us.es (M.A.C.-G.) \\ * Correspondence: abaratto@us.es
}

Received: 30 September 2018; Accepted: 13 November 2018; Published: 16 November 2018

\begin{abstract}
A feasibility study of an experimental setup for the irradiation of biological samples at the cyclotron facility installed at the National Centre of Accelerators (Seville, Spain) is presented. This cyclotron, which counts on an external beam line for interdisciplinary research purposes, produces an $18 \mathrm{MeV}$ proton beam, which is suitable for the irradiation of mono-layer cultures for the measurement of proton cell damages and Relative Biological Effectiveness (RBE) at energies below the beam nominal value. Measurements of this kind are of interest for proton therapy, since the variation of proton RBE at the distal edge of the Bragg curve may have implications in clinical proton therapy treatments. In the following, the characteristics of the beam line and the solutions implemented for the irradiation of biological samples are described. When dealing with the irradiation of cell cultures, low beam intensities and broad homogeneous irradiation fields are required, in order to assure that all the cells receive the same dose with a suitable dose rate. At the cyclotron, these constraints have been achieved by completely defocusing the beam, intercepting the beam path with tungsten scattering foils and varying the exit-window-to-sample distance. The properties of the proton beam thus obtained have been analysed and compared with Monte Carlo simulations. The results of this comparison, as well as the experimental measurement of the lateral dose profiles expected at the position of samples are presented. Meaningful dose rates of about 2-3 Gy/min have been obtained. Homogeneous lateral dose profiles, with maximum deviations of $5 \%$, have been measured at a distance of approximately $50 \mathrm{~cm}$ in air from the exit window, placing a tungsten scattering foil of $200 \mu \mathrm{m}$ in the beam path.
\end{abstract}

Keywords: proton beams; beam characterization; radiochromic films; radiobiology; dosimetry; IBA cyclotron

\section{Introduction}

Among charged hadron therapy techniques, proton therapy has become the most largely used thanks essentially to (1) its proved potential to better spare normal tissue if compared to conventional radiotherapy with photons (i.e., higher spatial dose conformity) and (2) the reduction of costs related to equipment acquisition and operation produced during the last decades, as compared to heavier ion beams. In addition, the dose delivered downstream the Bragg peak by proton beams is much lower than that delivered by heavier ion beams, due to the lower amount of nuclear fragments produced by protons along the beam path. 
Currently, in clinical practice, it is assumed that proton beams are $10 \%$ more efficient than clinical photon beams as for cell-killing effectiveness [1]; in other words, the Relative Biological Effectiveness (RBE) of clinical protons is considered to have a constant value of 1.1. The use of a constant RBE within every tissue and at each position of the spread-out Bragg peak of a proton beam is an approximation justified by the fact that the available biological data are insufficient to support the implementation of other proposed approaches in a clinical environment [2,3]. However, there are many works suggesting that proton RBE depends on various factors, including tissue type, biological endpoint, dose and radiation quality, and which varies towards the distal Bragg peak region, increasing with Linear Energy Transfer (LET) [4-6]. Furthermore, it has been claimed that ignoring the variations in RBE along the proton beam may have important clinical consequences [7], which make studies of RBE highly relevant.

Thus, radiobiological experiments with protons at energies typically found at the Bragg peak region of clinical beams (roughly below $40 \mathrm{MeV}$ ) are of interest. Ideally, such a work must be carried out at a facility providing proton beams with nominal energy below the limit mentioned, so that straggling due to passive degradation (widening the beam spectrum) is minimized. Frequently, these facilities were not projected for these type of experiments, thus optimization work is often needed [8-11].

At the National Centre of Accelerators (CNA, Seville, Spain), beam lines of this kind are available at the $3 \mathrm{MV}$ tandem (Pelletron 9SDH-2 model) and at the cyclotron (Cyclone 18/9 model) facilities, which can be adapted to allow reliable measurements and irradiations of mono-layer cell cultures at low proton energies, avoiding the inevitable uncertainties arising from the use of thick layers of degrading material. A setup for mono-layer cell culture irradiation at the tandem has already been proposed [12]. In this paper, the feasibility study of such a setup at the cyclotron is shown, focusing on the procedures to obtain the best irradiation conditions for radiobiological measurements and on the analysis of the beam characteristics by means of Monte Carlo simulations.

\section{Materials and Methods}

The cyclotron installed at the CNA in Seville is a Cyclone 18/9 from the IBA company (Ion Beam Applications, Louvain-La-Neuve, Belgium), which accelerates protons and deuterons up to 18 and $9 \mathrm{MeV}$, respectively. This cyclotron, mainly used for the production of short half-life radioisotopes for Positron Emission Tomography (PET), is equipped with an external beam line for interdisciplinary research. In the following, we provide comprehensive information about the proton beam achieved for the purpose of radiobiological measurements, following the characteristics of the experimental beam line and the solutions implemented to define a diagnostics scheme for the irradiation of biological samples.

\subsection{Beam Line}

The $18 \mathrm{MeV}$ proton beam, produced by the cyclotron, proceeds horizontally to the experimental room via the beam transport system, consisting of a variable graphite slit, an XY magnetic steerer, a quadrupole doublet and a quadrupole singlet. A two meter thick concrete wall, through which a stainless steel vacuum pipe transports the beam, separates the cyclotron bunker from the experimental room, where the beam is finally extracted in air. This section of the beam line can be isolated by means of a pneumatic valve and contains the following elements: a circular water-cooled Aluminium fixed collimator of $15 \mathrm{~mm}$ diameter, a pumping station and a retractable graphite Faraday cup for the measurement of current, covered with a phosphor scintillator (ZnS-Ag) to observe the beam shape and size by means of a video camera. Mounted on the exit flange, there is a $15 \mathrm{~mm}$ diameter graphite collimator, separated by one meter from the fixed collimator, to which a thin Mylar window is attached. A schematic representation of the beam line is shown in Figure 1. 


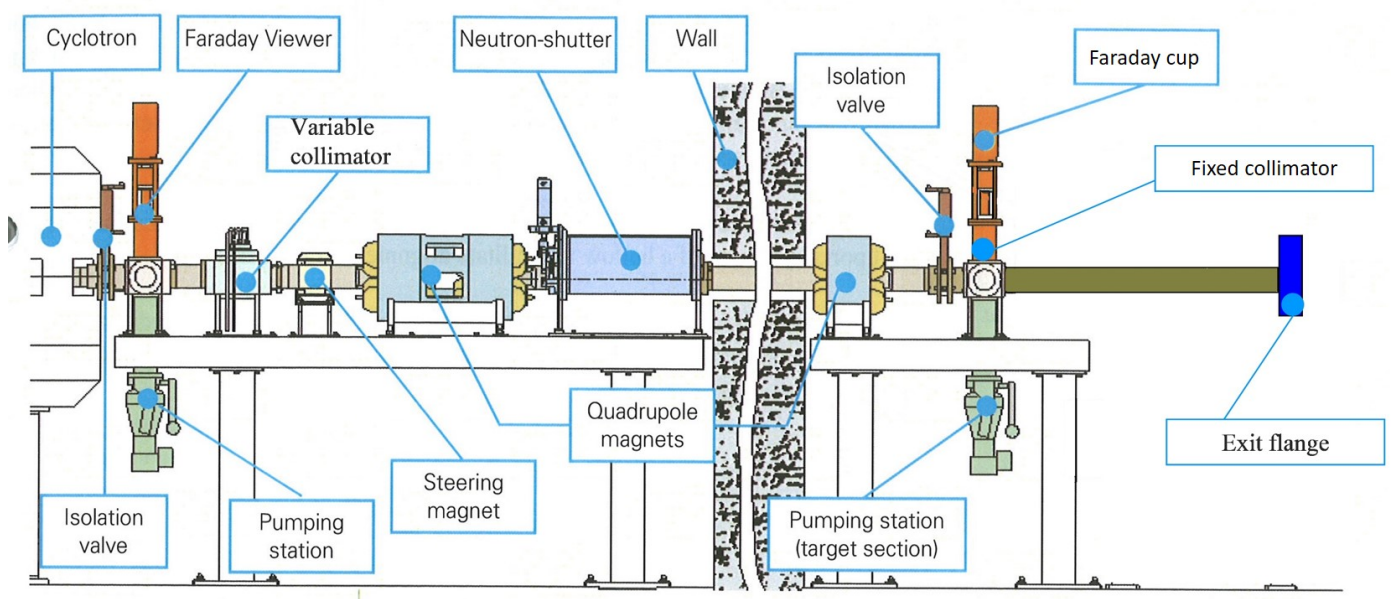

Figure 1. Layout of the external beam line.

Given the spatial configuration of the CNA cyclotron experimental beam line and the proton nominal energy, irradiation of cells should be performed with mono-layer cultures placed with vertical orientation in Petri dishes, as the range in water of the nominal proton beam is lower than $4 \mathrm{~mm}$. Different solutions can be implemented for the preparation and irradiation of the samples. On the one side, a system similar to the one described in [13] could be developed, with the seeded Petri dishes vertically inserted in a magazine filled with medium and extracted only for the time of irradiation. On the other side, customized Petri dishes, as those described in [14] or commercial ones, with an ultra-thin base for the cells to be attached, could be used, filling them with medium and irradiating them from behind. Following the characteristics of the cyclotron beam line, there are some major requirements to be taken into account when it comes to the irradiation of biological samples:

1. beam intensity of the order of tens of pA impinging on the sample, to control properly the fluence within suitable irradiation time scales;

2. broad irradiation field, of the order of few square centimetres to cover the whole sample area;

3. homogeneous beam profile over the sample surface, in both energy and spatial distributions.

To reach beam currents of the order of tens of $\mathrm{pA}$ on the sample, the beam intensity produced by the cyclotron has to be decreased. Therefore, the decision of using a completely defocused beam was made, turning off all the magnets for beam optics and lowering as much as possible the extracted beam current. With this arrangement, the beam current measured in the Faraday cup is of the order of hundreds of $\mathrm{pA}$, too high to meet the constraints for the irradiation of biological samples. Thus, the beam needs to be further scattered downstream the exit window. This, and the broadening of the irradiation field, is achieved by (1) placing tungsten foils of different thickness immediately after the exit window and (2) changing the amount of air between the window and the sample position.

\section{Beam Characteristics and Monte Carlo Simulations}

The cyclotron external beam line counts only on Faraday cups for beam diagnostics and, given the limited space available in the experimental room (approximately two meters from the exit window to the wall), the insertion of new elements for diagnostics extending the length of the beam line would not be easy. Furthermore, the necessity to turn off all the magnets, makes it difficult to control the beam optics and to determine the position of the maximum of the beam intensity. Therefore, information about the beam characteristics must be derived from measurements of beam profiles in air and compared with Monte Carlo simulations.

Firstly, the shape of the beam has been measured directly after the exit window, using Gafchromic EBT3 type films. As shown in Figure 2, the beam spot is approximately elliptical, with main axes of 
15 and $12 \mathrm{~mm}$ respectively, and the beam profile is asymmetric. These features are related to the inner characteristics of the beam coming from the cyclotron, since, without focusing elements, the beam presents some deviations with respect to the beam line axis. Measurements, however, are not affected by this initial non-uniformity because multiple Coulomb scattering happening both in air and in tungsten scattering foils blurs its effect at the sample position, approximately placed at a distance of $50 \mathrm{~cm}$ from the exit window.

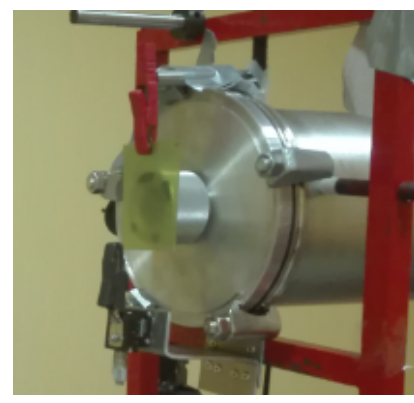

(a)

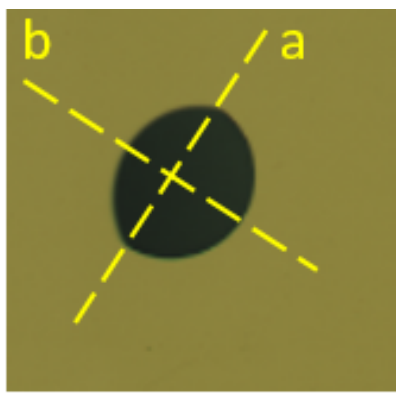

(b)

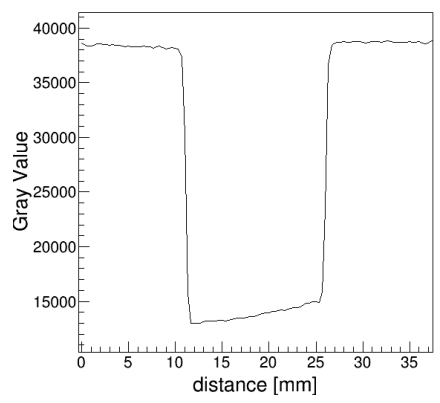

(c)

Figure 2. (a) EBT3 film mounted for the measurement of beam shape directly after the exit window; (b) EBT3 film showing beam shape; (c) profile of the beam taken along the "a" axis: the colour value of each pixel is plotted versus its position along the profile chosen. The lateral dimension of the beam at FWHM (Full Width at Half Maximum) is approximately equal to $15 \mathrm{~mm}$, which corresponds to the dimension of the graphite collimator.

Taking into account the shape of the beam shown in Figure 2 and considering the dimensions of the two collimators mounted inside the beam line and their distance, it is possible to infer the initial characteristics of the beam, to be implemented in Monte Carlo simulations and lately matched with experimental data [11]. Simulations have been performed with the SRIM (The Stopping and Range of Ions in Matter) code [15], using the SRIM Supporting Software Module [16] to produce a realistic proton beam. The energy of the beam has been set equal to the nominal one, $18 \mathrm{MeV}$, with a standard deviation of $1 \%$ from the mean value and Gaussian distribution. For the lateral shape of the beam and angular spread of the beam, uncorrelated Gaussian distributions with standard deviations of $5 \mathrm{~mm}$ and $6 \mathrm{mrad}$, respectively, have been considered.

\subsection{Experimental Setup}

The setup for the irradiation of biological samples, depicted in Figure 3, is similar to the one described in [12]. An external holder is placed at a distance of approximately $50 \mathrm{~cm}$ from the exit window, housing an ionization chamber (IC) to measure the charge produced by the proton beam. This chamber, fabricated at the GSI laboratory, is made of three thin parallel kapton electrodes, $7.5 \mu \mathrm{m}$ thick, with two air gaps of $6.75 \mathrm{~mm}$ thickness in between, operates at $400 \mathrm{~V}$ and is connected to a PTW electrometer for charge readout. The holder is made of two Polymethylmethacrylate (PMMA) supports, having each a circular collimator with a diameter of $35 \mathrm{~mm}$ and a thickness of $1 \mathrm{~cm}$; the ionization chamber is placed between them. The dimensions of these collimators have been chosen to fit the actual dimension of standard Petri dishes for cell culture, which can be mounted on the rear of the second PMMA support. This position is also used to measure beam profiles and make dosimetric studies by means of Gafchromic EBT3 type films. Finally, the whole setup is mounted on a moving table with millimetric precision, which can be moved remotely from the control room. To summarize, Table 1 collects a list of all the materials traversed by the proton beam and their thickness. 


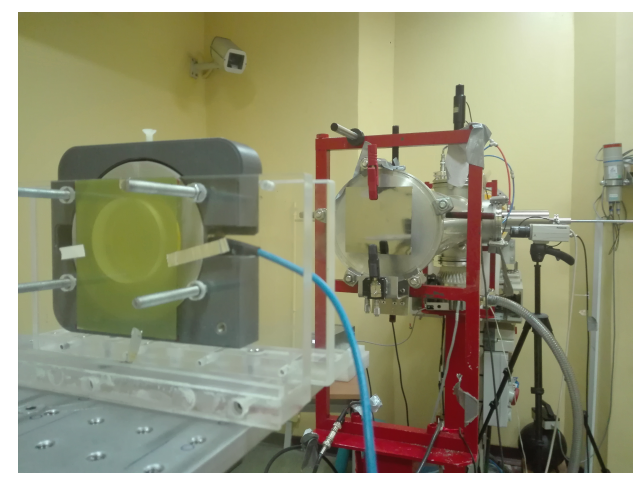

Figure 3. Picture of the experimental setup. An ionization chamber is placed between two PMMA supports on a moving table. A piece of Gafchromic EBT3 film is attached to the rear of the second PMMA support, after a circular collimator. The beam, coming from the front, traverses a tungsten scattering foil of variable thickness and approximately $50 \mathrm{~cm}$ of air, before reaching the ionization chamber.

Table 1. Material and thickness of the experimental setup components traversed by the proton beam. The column "order" indicates the sequence in which the layers of material are placed.

\begin{tabular}{cccc}
\hline Element & Material & Order & Thickness \\
\hline Exit window & Mylar & 1 & $125 \mu \mathrm{m}$ \\
Scattering Foil & Tungsten & 2 & $50-250 \mu \mathrm{m}$ \\
Window-sample distance & Air & 3 & $50 \mathrm{~cm}$ \\
IC-Electrode & Kapton & $4,6,8$ & $7.5 \mu \mathrm{m}$ \\
IC-Active volumes & Air & 5,7 & $6.75 \mathrm{~mm}$ \\
EBT3 film substrate & Polystyrene & 9,11 & $125 \mu \mathrm{m}$ \\
EBT3 film active layer & Lucite & 10 & $28 \mu \mathrm{m}$ \\
\hline
\end{tabular}

\subsection{Gafchromic EBT3 Films}

Radiochromic films, such as Gafchromic EBT3, are widely used for quality assurance measurements in radiation therapy, thanks to their high spatial resolution and their quasi water equivalence [17]. These films are mainly used for photon dosimetry because their response is independent on the energy of the incident clinical photons. This is not true in the case of proton irradiation, where an energy dependence emerges if the highly ionizing region of the Bragg peak falls inside the active layer of the film [18-20]. However, several studies have been done to evaluate the importance of this effect, and the use of Gafchromic films in proton dosimetry is well documented [21,22]. EBT3 type films are made of a single $28 \mu \mathrm{m}$ thick active layer, mounted between two $125 \mu \mathrm{m}$ thick polyester substrates. Under exposure to ionizing radiation, a polymerization reaction occurs within the active layer of the film, developing a blue colour dye. The subsequent change in optical density (OD) is correlated to the dose, and can be determined by a flatbed scanner. EBT3 films are self-developing films, and do not require post exposure processing. The full colour development of EBT3 films is usually very rapid, occurring in a few milliseconds. However, some chemical processes require more time, minutes and even hours, after irradiation, to reach completion. Therefore, a time window of $24 \mathrm{~h}$ between irradiation and scanning is usually recommended [23]. The film scanning must be performed in transmission mode, always in the same direction.

In this work, an Epson Perfection V700 photo scanner (Suwa, Nagano, Japan) has been used. Films have been loaded in 48-bit colour mode, without any image correction, and scan resolution of 75 dpi. Digitized films have been saved using uncompressed tagged image file format (tiff) to be lately analysed with Image (Image processing and analysis in Java), a public domain software for image processing.

The response of radiochromic films to irradiation is commonly expressed in terms of the net optical density (netOD), obtained for each RGB colour channel (Red, Green and Blue) as: 


$$
\text { netOD }=-\log _{10} \frac{I}{I_{0}}
$$

where $I$ is the colour value of an irradiated pixel and $I_{0}$ corresponds to a non-irradiated one. Among the three channels, the red colour channel presents the highest dose sensitivity.

\subsubsection{Calibration}

In order to relate the optical density to the dose deposited in the active layer of the EBT3 film, a calibration curve can be constructed. The dose deposited in the active layer $\left(D_{\mathrm{AL}}\right)$ of the film, can be evaluated from the proton fluence $\phi$ measured with the ionization chamber, by means of the following formula:

$$
D_{\mathrm{AL}}[\mathrm{Gy}]=1.6 \times 10^{-7} \cdot \frac{\mathrm{d} E}{\mathrm{~d} x}\left[\frac{\mathrm{MeV}}{\mathrm{cm}}\right] \cdot \phi\left[\mathrm{cm}^{-2}\right] \cdot \frac{1}{\rho}\left[\frac{\mathrm{cm}^{3}}{\mathrm{mg}}\right],
$$

where $\mathrm{d} E / \mathrm{d} x$ is the mean stopping power in the active layer material (Lucite), and $\rho$ is its density $\left(1.2 \mathrm{~g} / \mathrm{cm}^{3}\right)$. The proton fluence through the ionization chamber can be obtained from the charge output of the ionization chamber as follows:

$$
\phi\left[\mathrm{cm}^{-2}\right]=\frac{1}{A}\left[\mathrm{~cm}^{-2}\right] \cdot \frac{Q[\mathrm{nC}] \cdot W[\mathrm{eV}]}{e[\mathrm{nC}] \cdot E_{\mathrm{dep}}[\mathrm{eV}]},
$$

where $A$ is the area of the PMMA collimator placed upstream the ionization chamber, $Q$ is the charge collected by the ionization chamber and read with the electrometer, $W=34 \mathrm{eV}$ is the ionization potential of the gas contained in the active layers of the ionization chamber, $E_{\mathrm{dep}}$ is the mean energy deposited by individual protons in the ionization chamber and $e$ is the elementary charge in $\mathrm{nC}$. The computation of the dose is done under the assumption that the proton fluence does not vary in the area of the sample, for which the achievement of a homogeneous and uniform beam profile is essential. To compute $E_{\mathrm{dep}}$ in the ionization chamber and $\mathrm{d} E / \mathrm{d} x$ in the active layer of the film, the SRIM Monte Carlo code can be used, implementing the geometry of the experimental setup and setting the origin of the true beam immediately before the exit window.

\section{Results}

Following the method described in Section 2.3.1, a calibration curve has been built in the range 1-20 Gy. To this end, rectangular pieces of EBT3 film, cut from the same sheet and positioned with the same orientation with respect to the beam direction, were attached to the rear of the second PMMA support, immediately after the ionization chamber and the $35 \mathrm{~mm}$ diameter collimator (see Figure 3) and irradiated with different doses. Measurements were done at a distance of approximately $50 \mathrm{~cm}$ from the exit window with two different tungsten scattering foils: one of $150 \mu \mathrm{m}$ thickness, resulting in a mean kinetic energy of the proton beam at the sample position of $10.7 \mathrm{MeV}$, and the other of $200 \mu \mathrm{m}$ thickness, resulting in an mean kinetic energy at the sample position of $8.8 \mathrm{MeV}$. With the $200 \mu \mathrm{m}$ thickness scattering foil, only the range from 1 to $5 \mathrm{~Gy}$ was covered. Irradiations took from $10 \mathrm{~s}$ to $6 \mathrm{~min}$, with a dose rate of about $2-3 \mathrm{~Gy} / \mathrm{min}$.

As emerges from Figure $4 a$, the colour level of the irradiated area is not perfectly uniform, showing deviations from homogeneity that can be up to $10 \%$ in the worst cases. This factor has been taken into account in the calibration, where the largest uncertainty in the optical density of single data points derives from the inhomogeneity of the proton beam profile. To compute the optical density for each irradiated EBT3 piece, the mean color value inside a circular area (the same for every piece) of the irradiated region has been taken, and related to the colour value in the same area for a non-irradiated piece of the EBT3 film sheet, as shown in Figure 4b,c. 


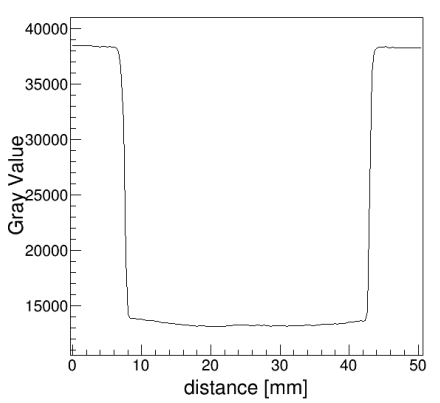

(a)

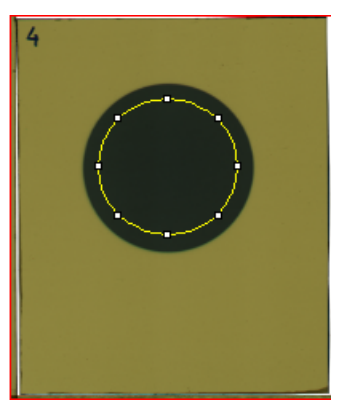

(b)

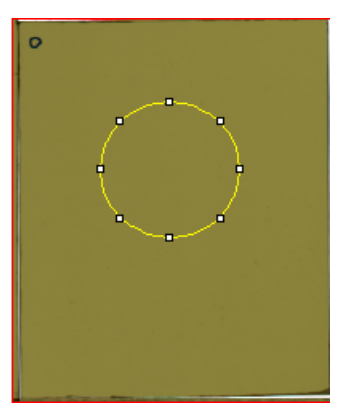

(c)

Figure 4. (a) colour level profile and (b) irradiated area of a piece of EBT3 film irradiated during approximately six minutes. The optical density associated with this piece of film is computed using Equation (1), where $I$ is the mean color value inside the yellow circular region in (b) and $I_{0}$ is the mean color value inside the same region in (c), which is a piece of non-irradiated film from the same sheet. A maximum deviation from the mean value of $4 \%$ is observed in the yellow region in (b).

The dose has been computed as stated in Section 2.3.1, using the SRIM Monte Carlo code to obtain the values of $E_{\mathrm{dep}}$ and $\mathrm{d} E / \mathrm{d} x$ to be used in Equations (3) and (2). The distributions of $E_{\mathrm{dep}}$ and the track-averaged distribution of $\mathrm{d} E / \rho \mathrm{d} x$, for the two energies considered, are shown in Figure 5 , while their mean values are listed in Table 2.

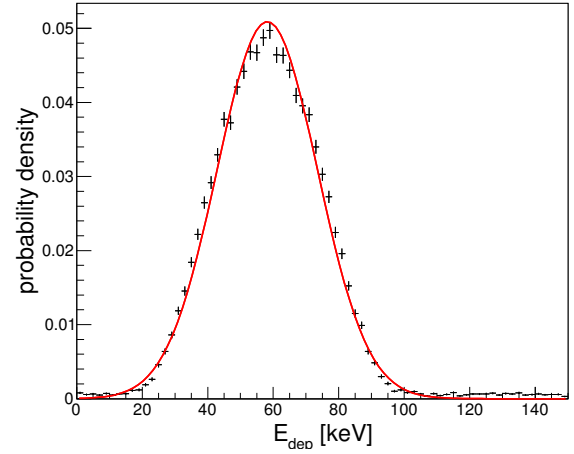

(a)

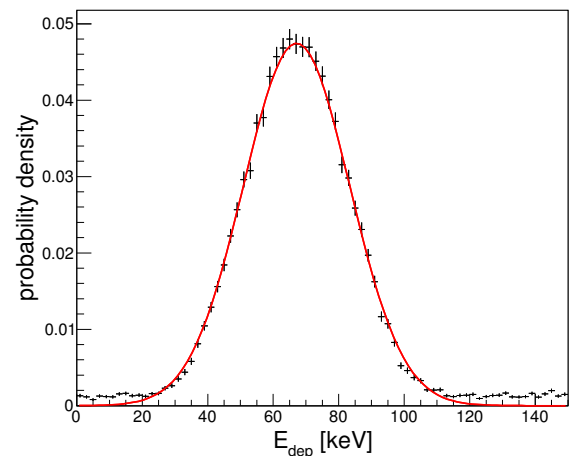

(c)

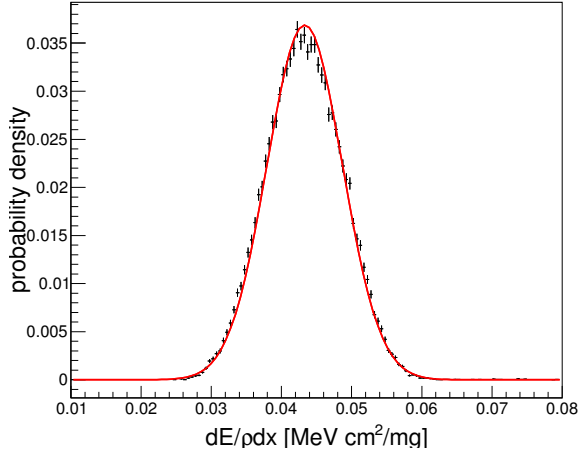

(b)

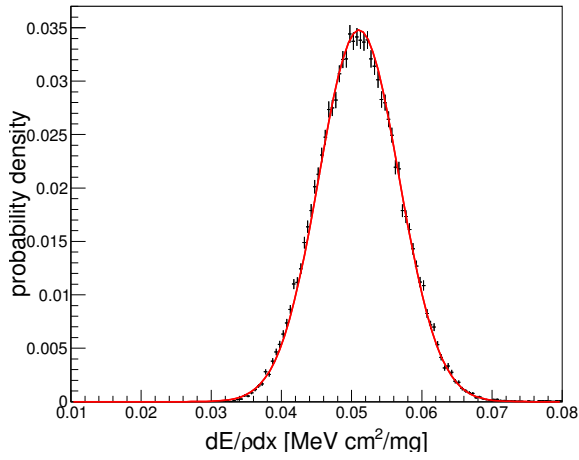

(d)

Figure 5. (a) distribution of energy deposited per individual proton track $\left(E_{\text {dep }}\right)$ in the ionization chamber and (b) track-averaged spectrum of $\mathrm{d} E / \rho \mathrm{d} x$ in the active layer of the film for the $10.7 \mathrm{MeV}$ proton beam; (c,d) are the same to $(\mathrm{a}, \mathrm{b})$ for the $8.8 \mathrm{MeV}$ proton beam. The red curves are the Gaussian fits of the simulation results (black markers). Mean values and uncertainties of the Gaussian fits are reported in Table 2. The referred proton beams were obtained after degradation in air and tungsten foils (see main text for details). 
Table 2. Mean values and associated errors obtained from a Gaussian fit of the distributions shown in Figure 5. Energy refers to the mean energy impinging on the active layer of the film, after degradation in tungsten foils and air. $E_{\mathrm{dep}}$ and $\mathrm{d} E / \rho \mathrm{d} x$ are, respectively, the mean energy deposited in the ionization chamber and the mean value of the track-averaged stopping power in the active layer of the film.

\begin{tabular}{ccc}
\hline $\begin{array}{c}\text { Energy of Protons } \\
\text { Impinging on the Active } \\
\text { Layer of the Film }\end{array}$ & $\begin{array}{c}\text { Energy Deposited in } \\
\text { the Ionization Chamber } \\
\left(\boldsymbol{E}_{\mathbf{d e p}}[\mathbf{k e V}]\right)\end{array}$ & $\begin{array}{c}\text { Track-Averaged Stopping Power } \\
\text { in the Active Layer of the Film } \\
\left(\mathbf{d} E / \rho \mathbf{d} \boldsymbol{x}\left[\mathbf{M e V c m}^{2} / \mathbf{m g}\right]\right)\end{array}$ \\
\hline $10.7 \mathrm{MeV}$ & $58.18 \pm 0.08$ & $(4.332 \pm 0.002) \times 10^{-2}$ \\
$8.8 \mathrm{MeV}$ & $67.1 \pm 0.1$ & $(5.105 \pm 0.003) \times 10^{-2}$ \\
\hline
\end{tabular}

The calibration curves for the three colour channels, plotting the dose against netOD, are shown in Figure 6. These curves have been fitted according to Equation (4), as suggested in literature [24],

$$
D=a \cdot \operatorname{netOD}+b \cdot \operatorname{netOD}^{c}
$$

where $a, b$, and $c$ are the fitting parameters; the second term accounts for the nonlinear nonlinearity of the dose response at high doses, and values of the parameter $c$ vary between 1 and 3 (see Table 3). As emerges from Figure 6, the response of the blue channel does not show a strong dose dependence, while responses in the red and green channel are highly dose dependent. In the following, only the red channel will be considered, as it presents the largest sensitivity against dose.

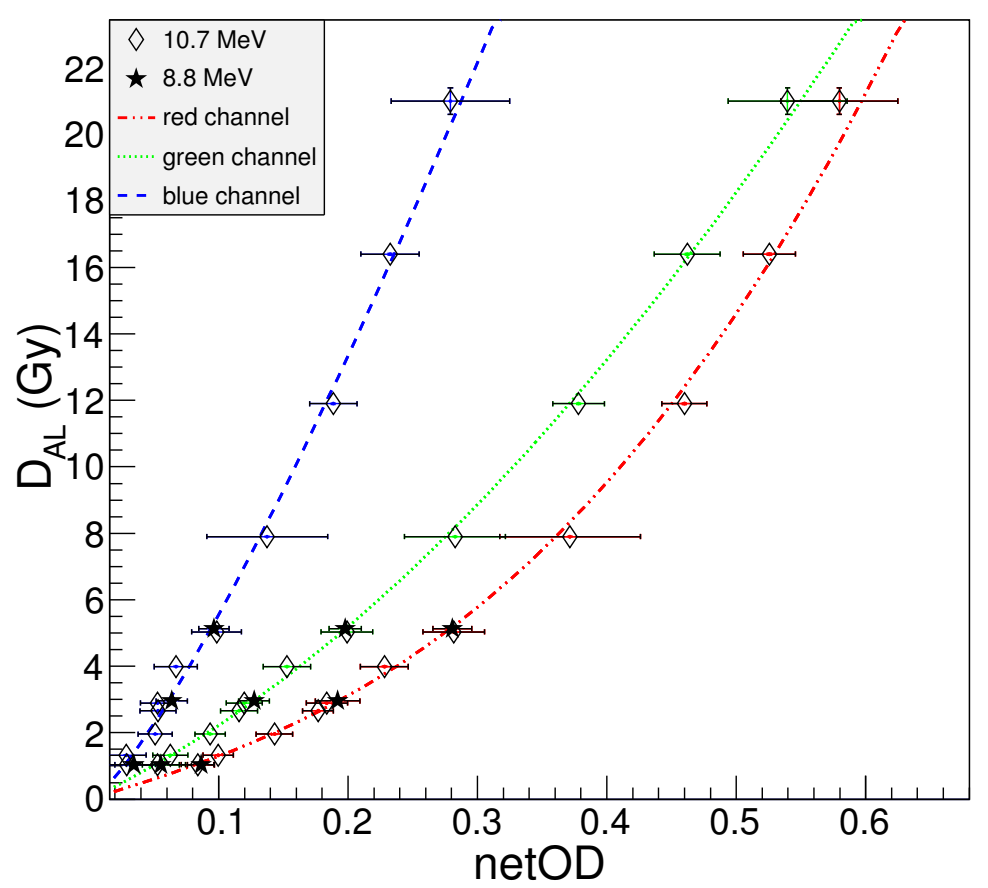

Figure 6. EBT3 calibration curves for two incident proton energies. The lines represent a fit to the two data sets, for each one of the colour channels.

Table 3. Results obtained for the parameters of Equation (4) from the fit of the data in Figure 6 for the three colour channels. For the blue channel, parameter $a$ has been fixed equal to zero.

\begin{tabular}{cccc}
\hline Parameter & Red Channel & Green Channel & Blue Channel \\
\hline$a$ [Gy] & $12 \pm 2$ & $18 \pm 7$ & 0 \\
$b$ [Gy] & $60 \pm 20$ & $40 \pm 10$ & $100 \pm 20$ \\
$c$ & $2.7 \pm 0.5$ & $1.9 \pm 0.8$ & $1.3 \pm 0.1$ \\
\hline
\end{tabular}


The calibration curve shown in Figure 6 can be used to scale colour profiles, measured in different conditions, to dose. This has been done to check the validity of the Monte Carlo simulation, using the following experimental configurations to irradiate full EBT3 films:

1. $48 \mathrm{~cm}$ of air between the exit window and the film, corresponding to a mean energy at the active volume of $15.6 \mathrm{MeV}$;

2. $63 \mathrm{~cm}$ of air between the exit window and the film, corresponding to a mean energy of $15.1 \mathrm{MeV}$;

3. $50 \mu \mathrm{m}$ thick tungsten foil attached to the exit window and $48 \mathrm{~cm}$ of air between the window and the film, corresponding to a mean energy of $14.2 \mathrm{MeV}$;

4. $50 \mu \mathrm{m}$ thick tungsten foil attached to the exit window and $63 \mathrm{~cm}$ of air between the window and the film, corresponding to a mean energy of $13.7 \mathrm{MeV}$;

The result of the comparison between the Monte Carlo simulation and the measured lateral dose profiles is shown in Figure 7. When no tungsten foil intercepts the beam path, the widths of Monte Carlo profiles are 5\% larger than measured ones. On the other hand, when the tungsten foils intercept the beam, Monte Carlo profiles appear to underestimate the width of the experimental ones, being around $12 \%$ smaller. As shown in Figure 8, no variation of the kinetic energy profile of the incident protons is expected off-axis, meaning that the $\mathrm{d} E / \rho \mathrm{d} x$ distribution can be considered to be the same for all the points in the same transverse plane.

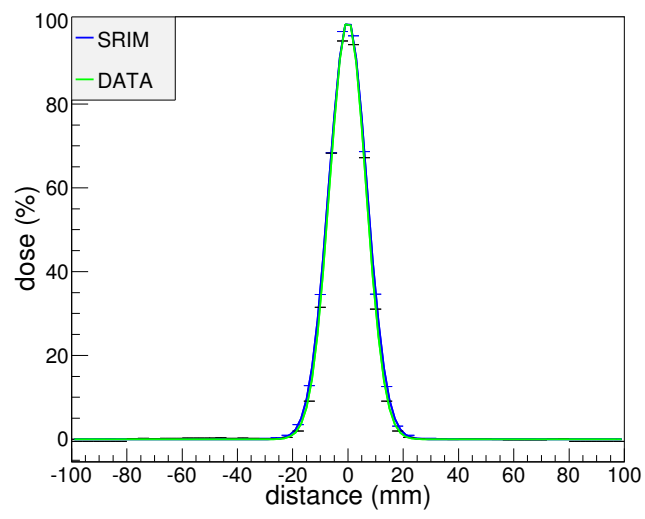

(a)

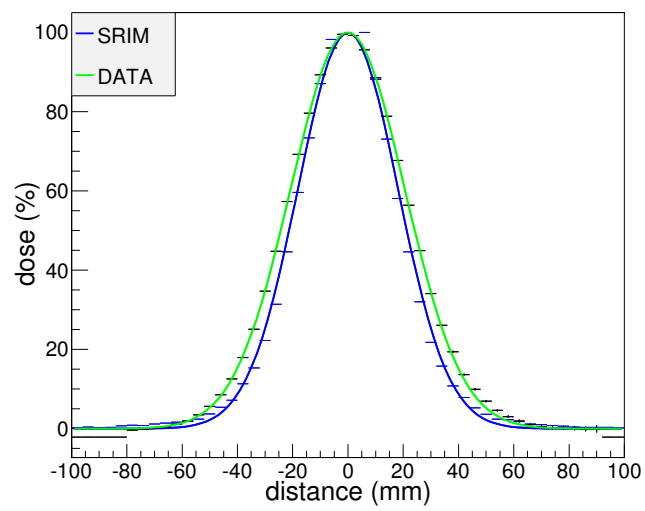

(c)

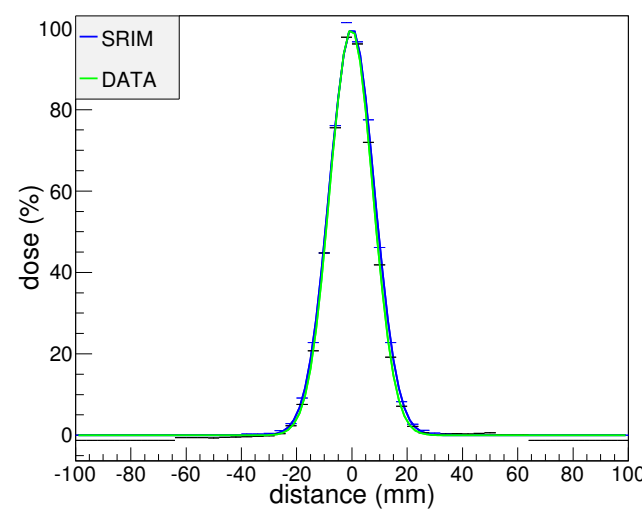

(b)

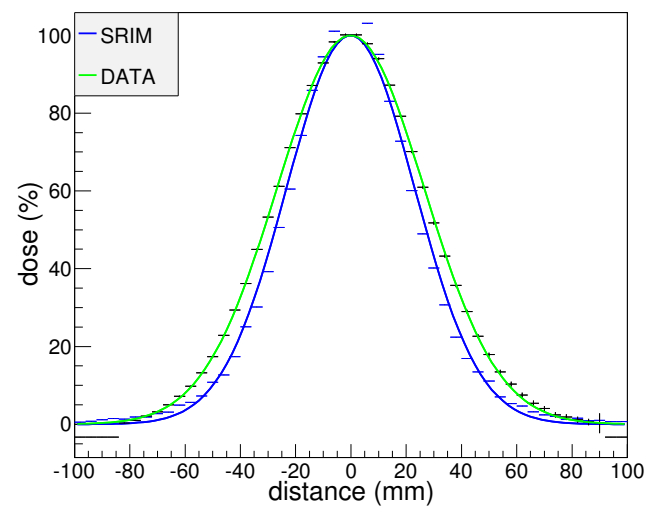

(d)

Figure 7. Lateral dose profiles measured (green) and simulated (blue) at different irradiation conditions; the energy value stands for the mean energy at the active volume of the EBT3 film. (a) $15.6 \mathrm{MeV}$ :

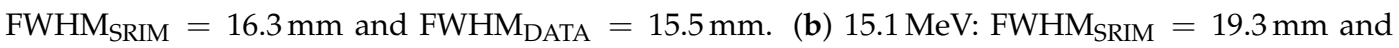

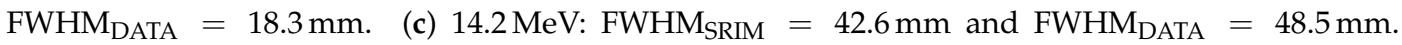
(d) $13.7 \mathrm{MeV}$ : FWHM $\mathrm{SRIM}=54.6 \mathrm{~mm}$ and $\mathrm{FWHM}_{\mathrm{DATA}}=62.9 \mathrm{~mm}$. 


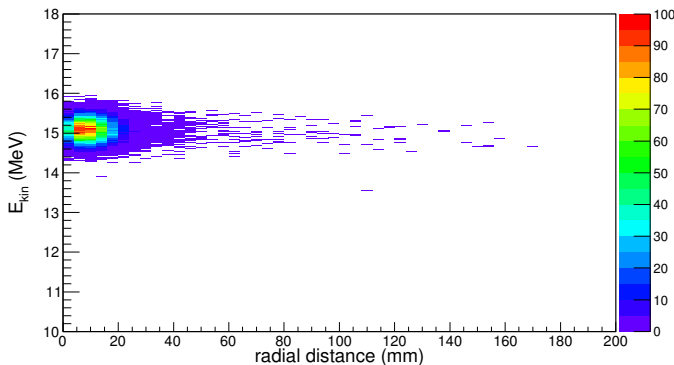

(a)

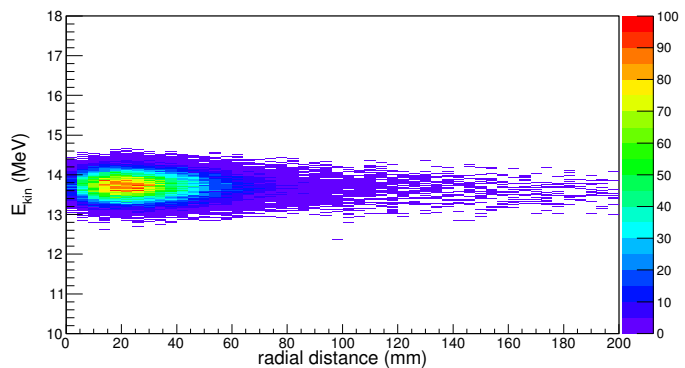

(b)

Figure 8. Example of simulated kinetic energy profile off-axis as a function of the radial distance, integrated over all directions contained in the scoring plane. The kinetic energy of the protons impinging on the active layer of the film is plotted against the distance of their interaction point from the beam axis in the transverse plane. Both plots represented here have been obtained for a distance between the exit window and the film of $63 \mathrm{~cm}$, without (a) and with (b) a $50 \mu \mathrm{m}$ tungsten scattering foil.

Finally, full lateral dose profiles have been measured at the position of the samples (approximately $50 \mathrm{~cm}$ from the exit window) with the $150 \mu \mathrm{m}$ and $200 \mu \mathrm{m}$ thick tungsten scattering foils, in order to obtain an evaluation of the deviations from dose homogeneity expectable during cell irradiations. An example of this profiles is shown in Figure 9a. Since cell samples are meant to be placed in Petri dishes of $35 \mathrm{~mm}$ diameter just after the PMMA collimator, a zoom of the profiles in the central $35 \mathrm{~mm}$ is plotted in Figure $9 \mathrm{~b}$. Maximum deviations from the central dose of $8 \%$ and $5 \%$ have been measured for the $150 \mu \mathrm{m}$ and $200 \mu \mathrm{m}$ thick tungsten scattering foils, respectively.

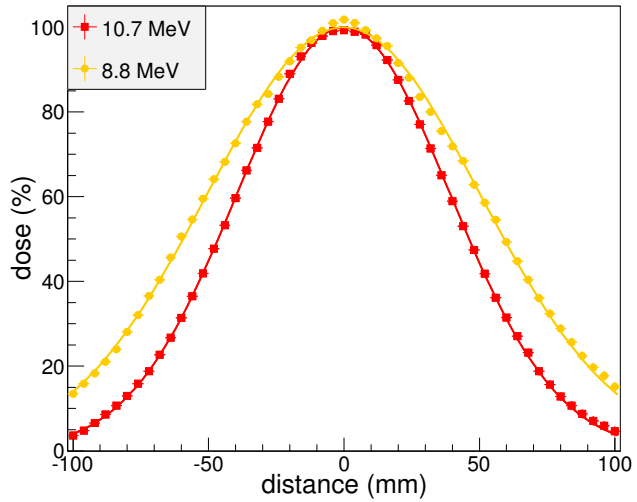

(a)

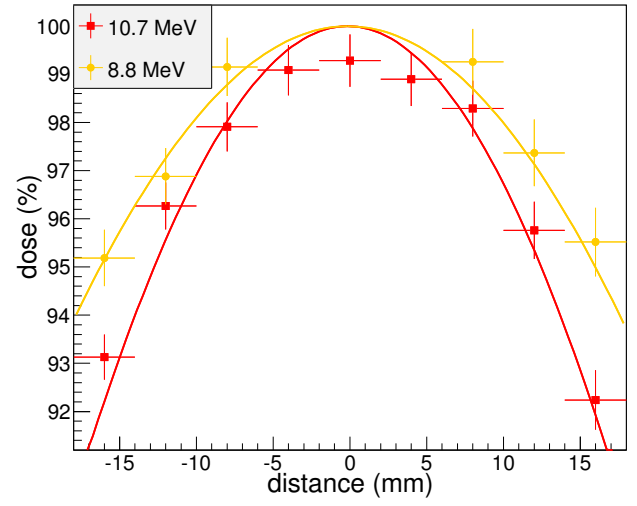

(b)

Figure 9. (a) comparison of full dose profiles obtained with a $150 \mu \mathrm{m}$ thick tungsten foil (red squares), corresponding to a mean energy of $10.7 \mathrm{MeV}$ at the film active volume, and with a $200 \mu \mathrm{m}$ thick foil (yellow dots), corresponding to a mean energy of $8.8 \mathrm{MeV}$; (b) zoom of the same profiles in the region where samples are meant to be placed, after the PMMA circular collimator of $35 \mathrm{~mm}$ diameter. Deviations from the maximum dose of $8 \%$ and $5 \%$ are observed for the $10.7 \mathrm{MeV}$ and $8.8 \mathrm{MeV}$ incident beams, respectively.

\section{Discussion}

An experimental setup for the irradiation of mono-layer cell samples at the $18 \mathrm{MeV}$ cyclotron facility installed at the CNA has been presented. The main advantage of using an $18 \mathrm{MeV}$ beam relies in the possibility of achieving proton energies corresponding to the Bragg peak region of a clinical proton beam with much lower energy spread. However, the range of applicability of such a beam is mostly limited to experiments with mono-layer cell cultures, for which the energy and the stopping power of 
protons reaching the cells can be calculated accurately. For floating cell cultures, one would have to take into account the beam energy degradation along the cell culture itself, introducing uncertainties due to the high variability of the stopping power in depth within the sample. Moreover, an additional source of uncertainty would arise from the imprecise knowledge of the position of the floating cells in the aqueous medium, which is important for an accurate calculation of the beam energy degradation.

The beam intensity has been decreased by lowering as much as possible the extraction current and by turning off all the magnets for beam optics. A further decrease in the beam intensity, as well as a broadening of the beam profile, has been achieved by intercepting the beam path with tungsten scattering foils of different thickness. An ionization chamber has been used to measure beam currents of the order of tens of pA in the position of cell sample (at a distance of approximately $50 \mathrm{~cm}$ from the exit window), corresponding to dose rates of about $2-3 \mathrm{~Gy} / \mathrm{min}$. Dose rates of this kind are clinically meaningful and highly recommended for radiobiological experiments [10].

The characteristics of the beam profile have been studied with Gafchromic EBT3 films in different irradiation conditions. As a first step, pieces of EBT3 film from the same sheet have been irradiated to various absorbed dose values and with different irradiation setups, one using a $150 \mu \mathrm{m}$ tungsten degrader, with which the proton beam irradiates the EBT3 active volume with a mean energy of 10.7 MeV, and another using a $200 \mu \mathrm{m}$ tungsten degrader, producing a mean energy of $8.8 \mathrm{MeV}$ at the same position. A calibration curve for EBT3 films, plotting the dose against the net optical density, has been constructed under the assumption of proton fluence homogeneity in the irradiated area. Deviations from homogeneity of the incident proton fluence have been accounted for in the horizontal error bars of the calibration curve (see Figure 6).

The calibration curve has been used to compare experimental profiles with simulated ones, in order to validate the Monte Carlo simulation and check the goodness of the initial beam parameters. Furthermore, a simulation has been done to assure that no variation of the beam energy profile, and therefore of the radiation quality, occurs at increasing distances from the beam axis. The simulation reproduces quite well experimental lateral dose profiles obtained without tungsten scattering foils intercepting the beam path, with differences around $5 \%$ in the width of the lateral dose distributions. However, for lower beam energies, when tungsten foils are inserted in the beam path, differences between Monte Carlo simulations and experimental results increase, the simulated widths $12 \%$ being smaller than the experimental ones. An explanation of this behaviour may be found in the error associated with the thickness of tungsten foils, which is around $10 \%$ as stated by the manufacturer. A study of the influence of this uncertainty in Monte Carlo simulations is currently ongoing. On the other hand, a systematic optimization of the initial beam parameters, as suggested in [11], could help improve Monte Carlo results. Finally, the source of the differences between Monte Carlo simulations and the experimental results may also arise from the physical models implemented in SRIM to reproduce lateral straggling. Therefore, Monte Carlo simulations with the Geant4 Monte Carlo toolkit [25-27] will be conducted, in order to compare the resulting outputs.

Full lateral dose profiles have been measured to evaluate the expected dose homogeneity with the proposed cell irradiation experimental conditions, which corresponds to a beam degraded with tungsten scattering foils of thickness equal or greater than $150 \mu \mathrm{m}$ and to a distance in air from the exit window of approximately $50 \mathrm{~cm}$. These conditions are necessary to ensure an irradiation field broad enough to cover the whole sample having a circular area of $35 \mathrm{~mm}$ diameter, and could be improved by inserting scattering foils inside the vacuum pipe, at a greater distance from the samples. However, maximum deviations of the order of $8 \%$ have already been achieved for the worst case (150 $\mu \mathrm{m}$ thickness), a result which is comparable with the ones obtained for the same purpose at similar facilities [8,9,11,12]. 
Author Contributions: Conceptualization, A.B.-R., M.C.J.-R., M.A.C.-G., J.M.E. and M.I.G.; Methodology, Cristina Battaglia, A.B.-R., M.C.J.-R., M.A.C.-G. and J.M.E.; Software, A.B.-R. and M.A.C.-G.; Formal Analysis, A.B.-R., M.A.C.-G. and M.C.J.-R.; Investigation, A.B.-R., M.C.J.-R., Cristina Battaglia, J.G.-L., M.I.G., M.A.C.-G. and J.M.E.; Resources, M.C.J.-R., J.G.-L. and J.M.E.; Data Curation, A.B.-R. and M.C.J.-R.; Writing-Original Draft Preparation, A.B.-R. and M.A.C.-G.; Writing-Review and Editing, A.B.-R., M.C.J.-R., M.C.B., M.I.G., M.A.C.-G. and J.M.E.; Supervision, M.C.J.-R., M.A.C.-G. and J.M.E.; Project Administration, M.A.C.-G. and J.M.E.; Funding Acquisition, J.M.E. and M.I.G.

Funding: This project has received funding from the European Union's Horizon 2020 research and innovation programme under the Marie Sklodowska-Curie Grant No. 675265, OMA-Optimization of Medical Accelerators, from the Spanish Ministry of Economy and Competitiveness under Grant No. FPA2016-77689-C2-1-R, and from the Council for Innovation, Science and Commerce of the Andalusian Government under Grant No. P12-FQM-1605.

Conflicts of Interest: The authors declare no conflict of interest.

\section{References}

1. International Commision on Radiation Units and Measurements. Prescribing, Recording and Reporting Proton-Beam Therapy, (ICRU Report 78). J. ICRU 2007, 2, 1-210.

2. Giovannini, G.; Böhlen, T.; Cabal, G.; Bauer, J.; Tessonnier, T.; Frey, K.; Debus, J.; Mairani, A.; Parodi, K. Variable RBE in proton therapy: Comparison of different model predictions and their influence on clinical like-scenarios. Radiat. Oncol. 2016, 11, 68. [CrossRef] [PubMed]

3. Carabe, A.; Moteabbed, M.; Depauw, N.; Schuemann, J.; Paganetti, H. Range uncertainty in proton therapy due to variable biological effectiveness. Phys. Med. Biol. 2012, 57, R1159-R1172. [CrossRef] [PubMed]

4. Paganetti, H.; Niemierko, A.; Ancukiewicz, M.; Gerweck, L.E.; Goitein, M.; Loeffler, J.S.; Suit, H.D. Relative biological effectiveness (RBE) values for proton beam therapy. Int. J. Radiat. Oncol. Biol. Phys. 2002, 53, 407-421. [CrossRef]

5. Matsuura, T.; Egashira, Y.; Nishio, T.; Matsumoto, Y.; Wada, M.; Koike, S.; Furusawa, Y.; Kohno, R.; Nishioka, S.; Kameoka, S.; et al. Apparent absence of proton beam dose rate effect and possible differences in RBE between Bragg peak and plateau. Med. Phys. 2010, 37, 5376-5381. [CrossRef] [PubMed]

6. Paganetti, H. Relative biological effectiveness (RBE) values for proton beam therapy. Variations as a function of biological endpoint, dose and linear energy transfer. Phys. Med. Biol. 2014, 59, R419-R472. [CrossRef] [PubMed]

7. Wedenberg, M.; Toma-Dasu, I. Disregarding RBE variation in treatment plan comparison may lead to bias in favor of proton plans. Med. Phys. 2014, 41, 091706. [CrossRef] [PubMed]

8. Belli, M.; Cherubini, R.; Galeazzi, G.; Mazzucato, S.; Moschini, G.; Sapora, O.; Simone, G.; Tabocchini, M.A. Proton irradiation facility for radiobiological studies at a $7 \mathrm{MV}$ Van de Graaf accelerator. Nucl. Instrum. Meth. Phys. Res. A 1987, 256, 576-580. [CrossRef]

9. Scampoli, P.; Casale, M.; Durante, M.; Grossi, G.; Pugliese, M.; Gialanella, G. Low-energy light ion irradiation beam-line for radiobiological studies. Nucl. Instrum. Meth. Phys. Res. B 2001, 174, 337-343. [CrossRef]

10. Huerta-Parajon, M.; Martinez-Ballarin, R.; Abad, E. Design study of the ESS-Bilbao $50 \mathrm{MeV}$ proton beam line for radiobiological studies. Nucl. Instrum. Meth. Phys. Res. B 2015, 344, 33-38. [CrossRef]

11. Tordis, J.D.; Rykkelid, A.M.; Stokkevåg, C.H.; Mairani, A.; Görgen, A.; Edin, N.J.; Rørvik, E.; Fjæra, L.F.; Malinen, E.; Ytre-Hauge, K.S. Monte Carlo simulations of a low energy proton beamline for radiobiological experiments. Acta Oncol. 2017, 56, 779-786. [CrossRef]

12. Battaglia, M.C.; Schardt, D.; Espino, J.M.; Gallardo, M.I.; Cortés-Giraldo, M.A.; Quesada, J.M.; Lallena, A.M.; Miras, H.; Guirado, D. Dosimetric response of radiochromic films to protons of low energies in the Bragg peak region. Phys. Rev. Accel. Beams 2016, 19, 064701. [CrossRef]

13. Kraft-Weyrather, W.; Kraft, G.; Ritter, S.; Scholz, M.; Stanton, J.A. The preparation of biological targets for heavy-ion experiments up to $20 \mathrm{MeV} / \mathrm{u}$. Nucl. Instrum. Meth. Phys. Res. A 1989, 282, 22-27. [CrossRef]

14. Belli, M.; Cherubini, R.; Finotto, S.; Moschini, G.; Sapora, O.; Simone, G.; Tabocchini, M.A. RBE-LET Relationship for the Survival of V79 Cells Irradiated with Low Energy Protons. Int. J. Radiat. Biol. 1989, 55, 93-104. [CrossRef] [PubMed]

15. Ziegler, J.F.; Ziegler, M.D.; Biersack, J.P. SRIM-The stopping and range of ions in matter. Nucl. Instrum. Meth. Phys. Res. B 2010, 268, 1818-1823. [CrossRef]

16. Pavlovič, M.; Strašík, I. Supporting routines for the SRIM code. Nucl. Instrum. Meth. Phys. Res. B 2007, 257, 601-604. [CrossRef] 
17. Devic, S. Radiochromic film dosimetry: Past, present and future. Phys. Med. 2011, 27, 122-134. [CrossRef] [PubMed]

18. Piermattei, A.; Miceli, R.; Azario, L.; Fidanzio, A.; Delle Canne, S.; De Angelis, C.; Onori, S.; Pacilio, M.; Petetti, E.; Raffaele, L.; et al. Radiochromic film dosimetry of a low energy proton beam. Med. Phys. 2000, 27, 1655-1660. [CrossRef] [PubMed]

19. Zhao, L.; Das, I.J. Gafchromic EBT film dosimetry in proton beams. Phys. Med. Biol. 2010, 55, N291-N301. [CrossRef] [PubMed]

20. Kirby, D.; Green, S.; Palmans, H.; Hugtenburg, R.; Wojnecki, C.; Parker, D. LET dependence of GafChromic films and an ion chamber in low-energy proton dosimetry. Phys. Med. Biol. 2010, 55, 417-433. [CrossRef] [PubMed]

21. Sorriaux, J.; Kacperek, A.; Rossomme, S.; Lee, J.A.; Bertrand, D.; Vynckier, S.; Sterpin, E. Evaluation of Gafchromic EBT3 films characteristics in therapy photon, electron and proton beams. Phys. Med. 2013, 29, 599-606. [CrossRef] [PubMed]

22. Reinhardt, S.; Würl, M.; Greubel, C.; Humble, N.; Wilkens, J.J.; Hillbrand, M.; Mairani, A.; Assmann, W.; Parodi, K. Investigation of EBT2 and EBT3 films for proton dosimetry in the 4-20 MeV energy range. Radiat. Environ. Biophys. 2015, 54, 71-79. [CrossRef] [PubMed]

23. Niroomand-Rad, A.; Blackwell, C.R.; Coursey, B.M.; Gall, K.P.; Galvin, J.M.; McLaughlin, W.L.; Meigooni, A.S.; Nath, R.; Rodgers, J.E.; Soares, C.G. Radiochromic film dosimetry: Recommendations of AAPM Radiation Therapy Committee Task Group 55. Med. Phys. 1998, 25, 2093-2115. [CrossRef] [PubMed]

24. Devic, S.; Seuntjens, J.; Hegyi, G.; Podgorsak, E.B.; Soares, C.G.; Kirov, A.S.; Ali, I.; Williamson, J.F.; Elizondo, A. Dosimetric properties of improved GafChromic films for seven different digitizers. Med. Phys. 2004, 31, $2392-2401$. [CrossRef] [PubMed]

25. Agostinelli, S.; Allison, J.; Amako K.; Apostolakis, J.; Araujo, H.; Arce, P.; Asai, M.; Axen, D.; Banerjee, S.; Barrand, G.; et al. Geant4-A simulation toolkit. Nucl. Instrum. Meth. Phys. Res. A 2003, 31, 861-874. [CrossRef]

26. Allison, J.; Amako, K.; Apostolakis, J.; Araujo, H.; Arce Dubois, P.; Asai, M.; Barrand, G.; Capra, R.; Chauvie, S.; Chytracek, R.; et al. Geant4 developments and applications. IEEE Trans. Nucl. Sci 2006, 53, 270-278. [CrossRef]

27. Allison, J.; Amako, K.; Apostolakis, J.; Arce, P.; Asai, M.; Aso, T.; Bagli, E.; Bagulya, A.; Banerjee, S.; Barrand, G.; et al. Geant4-A simulation toolkit. Nucl. Instrum. Meth. Phys. Res. A 2016, 835, 186-225. [CrossRef]

(C) 2018 by the authors. Licensee MDPI, Basel, Switzerland. This article is an open access article distributed under the terms and conditions of the Creative Commons Attribution (CC BY) license (http://creativecommons.org/licenses/by/4.0/). 